\title{
Strain-dependent magnetic configurations in manganite-titanate heterostructures probed with soft X-ray techniques ${ }^{\star}$
}

\author{
R.V. Chopdekar ${ }^{1, a}$, J. Heidler ${ }^{1}$, C. Piamonteze ${ }^{1}$, Y. Takamura ${ }^{2}$, A. Scholl ${ }^{3}$, S. Rusponi ${ }^{4}$, H. Brune ${ }^{4}$, \\ L.J. Heyderman ${ }^{1}$, and F. Nolting ${ }^{1}$ \\ 1 Paul Scherrer Institut, 5232 Villigen PSI, Switzerland \\ 2 Department of Chemical Engineering and Materials Science, University of California, CA 95616 Davis, USA \\ 3 Advanced Light Source, Lawrence Berkeley National Laboratory, CA 94720 Berkeley, USA \\ 4 Institute of Condensed Matter Physics, École Polytechnique Fédérale de Lausanne, 1015 Lausanne, Switzerland
}

Received 31 October 2012 / Received in final form 11 February 2013

Published online 3 June 2013 - (c) EDP Sciences, Società Italiana di Fisica, Springer-Verlag 2013

\begin{abstract}
We present a detailed study on the strain-induced magnetic domain structure of a $(\mathrm{La}, \mathrm{Sr}) \mathrm{MnO}_{3}$ thin film epitaxially grown on a $\mathrm{BaTiO}_{3}$ substrate through the use of polarization-dependent X-ray photoemission electron microscopy and X-ray absorption spectroscopy. Angular-dependent measurements allow us to detect vector magnetization on a single-domain scale, and we relate the strain-induced changes in magnetic anisotropy of the ferromagnetic film to the ferroelectric domain structure of the underlying substrate using X-ray magnetic circular and linear dichroism spectro-microscopy. Comparisons to measurements on a nearly strain free film of $(\mathrm{La}, \mathrm{Sr}) \mathrm{MnO}_{3}$ grown on a $(\mathrm{La}, \mathrm{Sr})(\mathrm{Al}, \mathrm{Ta}) \mathrm{O}_{3}$ substrate illustrate that the $\mathrm{BaTiO}_{3}$ ferroelectric domain structure imprints specific domain sizes and wall orientations in the $(\mathrm{La}, \mathrm{Sr}) \mathrm{MnO}_{3} / \mathrm{BaTiO}_{3}$ artificial multiferroic heterostructure. Furthermore, a change of the $\mathrm{BaTiO}_{3}$ ferroelectric domain structure either with temperature or with applied electric field results in a corresponding change in the ( $\mathrm{La}, \mathrm{Sr}) \mathrm{MnO}_{3}$ ferromagnetic domain structure, thus showing a possible route to obtain room-temperature electric field control of magnetic anisotropy at the nanoscale.
\end{abstract}

\section{Introduction}

Recent studies of artificial multiferroic heterostructures consisting of epitaxial ferromagnetic and ferroelectric layers have shown large structural, electronic and magnetic changes in the ferromagnetic layer induced by the ferroelectric layer, illustrating that such artificial systems can be a promising route to obtaining room temperature magneto-electric coupling in nanoscale systems [1]. Spinel-structure ferromagnetic thin films have commonly been used in such artificial multiferroics, but they exhibit myriad defects such as anti-phase boundaries at the film substrate interface [2], which can reduce the efficacy of the magneto-electric coupling. To avoid such defects, coupling in $(\mathrm{La}, \mathrm{Sr}) \mathrm{MnO}_{3}-\mathrm{BaTiO}_{3}$ (LSMO-BTO) composite multiferroics has been explored as a function of temperature as well as applied magnetic and electric field.

It is well-known that epitaxial strain can have a large influence on the Curie temperature and magnetic anisotropy of LSMO thin films [3], thus by using a ferroelectric substrate such as BTO, one could try to dynamically

\footnotetext{
* Contribution to the Topical Issue "New Trends in Magnetism and Magnetic Materials", edited by Francesca Casoli, Massimo Solzi and Paola Tiberto.

a e-mail: Rajesh.Chopdekar@psi.ch
}

tune the magnetic properties of LSMO. Indeed, this material system shows coincident changes in LSMO resistivity, magnetization and lattice parameter at phase transitions of the BTO substrate $[4,5]$. Furthermore, an applied electric field across a BTO substrate, which changes the relative populations of a- and c-type ferroelectric domains, can induce persistent and sharp changes in LSMO magnetization [6].

While studies have shown that significant magnetoelectric coupling is possible in the LSMO-BTO system, such results are spatially averaged over macroscopic distances. Thus, for implementation of such materials at technologically relevant length scales, we need a comprehensive understanding of artificial multiferroic properties on the sub-micron length scale (i.e. the length scale of single ferroelectric and ferromagnetic domains). With X-ray photoemission electron microscopy (PEEM), we have shown that an artificial multiferroic system of a spinel magnetostrictive film grown on a perovskite piezoelectric substrate can be studied on the length scale of individual ferroic domains, and that the ferroelectric domain structure can imprint domain structures in the ferrimagnetic phase of the artificial multiferroic [7]. Additionally, X-ray spectroscopy has been shown to offer great insight into the influence of epitaxial strain sign and magnitude, as well 
as interfacial or surface effects on the ferromagnetic order of thin LSMO films [8-10].

In this work, we explore magneto-electric coupling in the isostructural LSMO-BTO system on varying the temperature or applying an electric field by using polarizationdependent X-ray absorption spectroscopy and spectromicroscopy. We have used substrate-induced strain to strongly affect the domain structure of LSMO films as seen by the strong difference in anisotropy of LSMO coupled to a- and c-type BTO domains. Temperature dependent measurements elucidate the connection between the BTO ferroelectric domain structure and magnetic anisotropy of the LSMO film, and we demonstrate that electric field pulses can induce the rotation of the LSMO magnetization direction at room temperature.

\section{Experimental}

A series of $29 \mathrm{~nm}$ and $53 \mathrm{~nm}$ thick LSMO films near the optimal doping concentration $\left(\mathrm{La}_{0.7} \mathrm{Sr}_{0.3} \mathrm{MnO}_{3}\right)$ were grown on $(001)$ oriented $(\mathrm{La}, \mathrm{Sr})(\mathrm{Al}, \mathrm{Ta}) \mathrm{O}_{3}$ (LSAT) and (001) oriented BTO substrates by pulsed laser deposition. Typical deposition conditions $\left(700{ }^{\circ} \mathrm{C}\right.$ substrate temperature, 300 mTorr $\mathrm{O}_{2}$ partial pressure, $1 \mathrm{~J} / \mathrm{cm}^{2}$ laser fluence) for obtaining low roughness LSMO films on (001) $\mathrm{SrTiO}_{3}$ [11] were also found to produce films with low roughness on LSAT and BTO substrates. The films were cooled to $500{ }^{\circ} \mathrm{C}$ in $300 \mathrm{~m}$ Torr $\mathrm{O}_{2}$, then annealed for $20 \mathrm{~min}$ in $0.5 \mathrm{~atm} \mathrm{O}_{2}$ pressure before cooling to room temperature to reduce the density of oxygen vacancies in the film. In order to measure any electric field-induced changes in the film magnetic domain structure, BTO substrates were mechanically thinned from the back to approximately $0.2 \mathrm{~mm}$ after LSMO deposition, and $5 \mathrm{~nm} \mathrm{Cr} / 50 \mathrm{~nm} \mathrm{Au}$ contacts were placed on the back of the thinned BTO substrates.

$\mathrm{X}$-ray PEEM experiments were performed in $16^{\circ}$ grazing incidence at the surface/interface: microscopy (SIM) beamline [12] at the Swiss Light Source and in $30^{\circ}$ grazing incidence at the PEEM3 microscope of the Advanced Light Source. Samples were measured in a demagnetized state; the demagnetizing protocol is described elsewhere[7]. Room temperature total electron yield spectroscopy measurements were performed at normal incidence (X-ray propagation direction along the sample normal) in a field of up to $2 \mathrm{~T}$ applied in the sample plane at the X-Treme beamline of the Swiss Light Source [13]. It should be noted that the field was tilted out of the sample plane by $8^{\circ}$ to enhance electron yield. Samples were measured at the $\mathrm{Mn} \mathrm{L}_{3,2}$ edges (approximately 642 and $653 \mathrm{eV}$ ) using greater than $97 \%$ linearly polarized and $97 \%$ circularly polarized X-rays in the temperature range of $300 \mathrm{~K}$ to $580 \mathrm{~K}$ at the SIM beamline microscope. Additionally, measurements in the temperature range of $160 \mathrm{~K}$ to $300 \mathrm{~K}$ were taken at the PEEM3 microscope. X-ray magnetic circular dichroism (XMCD), i.e. the difference in X-ray absorption between left and right circularly polarized X-rays, was utilized to probe the ferromagnetic properties of the LSMO films, whereas X-ray linear dichroism (XLD) relies on the difference in absorption between horizontally and vertically polarized X-rays and is sensitive to electronic anisotropy in LSMO originating from sources such as ferromagnetic order and structural anisotropy [14].

\section{Results}

\subsection{Influence of epitaxial strain on LSMO magnetic domains}

We may evaluate the impact of BTO ferroelectric domain structure on the magnetic domain structure of LSMO by comparing nearly-strain free films grown on LSAT with those grown under tensile strain on BTO. A c/a ratio of 0.983 with a pseudocubic unit cell volume of $58.55 \AA^{3}$ was extracted from hard X-ray diffraction reciprocal space mapping of the 002 and 303 reflections of a $53 \mathrm{~nm}$ LSMO film on a polydomain BTO substrate. The averaged strain of the film is in biaxial tension, but the local strain state depends on the orientation of each BTO ferroelectric domain at the film-substrate interface. In the tetragonal phase of BTO an $a$-axis ferroelectric domain, whose ferroelectric moment is in the plane of the substrate along a pseudocubic $\langle 100\rangle$ direction, imposes a uniaxial modulation of the strain, whereas a $c$-axis ferroelectric domain imposes a biaxially symmetric strain.

The strain has a marked impact on the magnetic domain structure as can be seen in the PEEM images of Figure 1. The PEEM XMCD image of the LSMO film on LSAT has four levels of contrast, signifying four orientations of ferromagnetic domains, labeled A, B, C, and D, evenly distributed across the field of view in Figure 1a. As the level of contrast is proportional to the projection of magnetization along the X-ray propagation direction, we can infer that the LSAT sample has domains pointing along four equivalent $\langle 110\rangle$ in-plane directions. We can confirm the in-plane nature of the domains by performing an azimuthal scan of the sample, which shows that each domain has a contrast reversal upon $180^{\circ}$ rotation of the sample, and the maximum level of contrast is equivalent for each set of domains. In comparison, the domain structure of LSMO on BTO in Figure 1b is heterogeneous, with some regions showing bright and dark contrast and a central stripe showing a neutral contrast. Across the sample, borders between regions of different contrast can be oriented along either $\langle 100\rangle$ or $\langle 110\rangle$ families of directions, and in the case of Figure 1b, the stripe is oriented along a $\langle 110\rangle$ type direction.

The 3D magnetization profile of a given field of view can be extracted from images taken at 3 or more azimuthal sample angles. An example of this vector decomposition for the $29 \mathrm{~nm}$ thick LSMO/BTO sample is shown in Figure 2 , which is obtained by fitting the contrast intensity in a point-by-point manner from 5 images at azimuthal angles between $0-180^{\circ}$, and this analysis reveals that the heterogeneous contrast of Figure $1 \mathrm{~b}$ is due to regions whose magnetic easy axes lie along alternating [100] and [010] directions. The borders between regions are along a $\langle 110\rangle$ type direction, and our previous study has shown that this configuration of ferromagnetic domains is due to the 
(a)

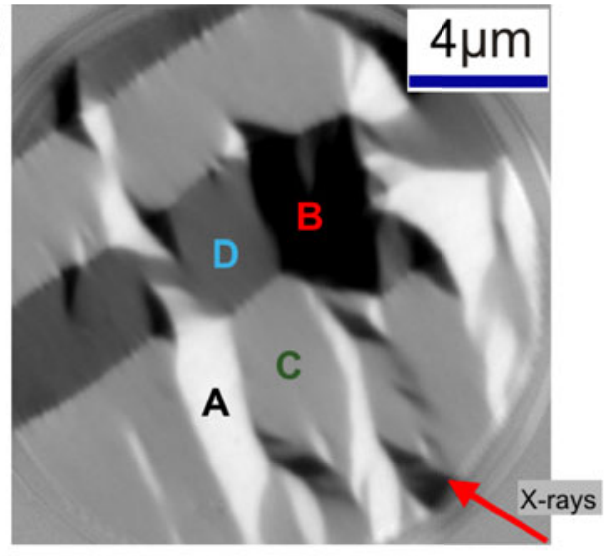

(b)

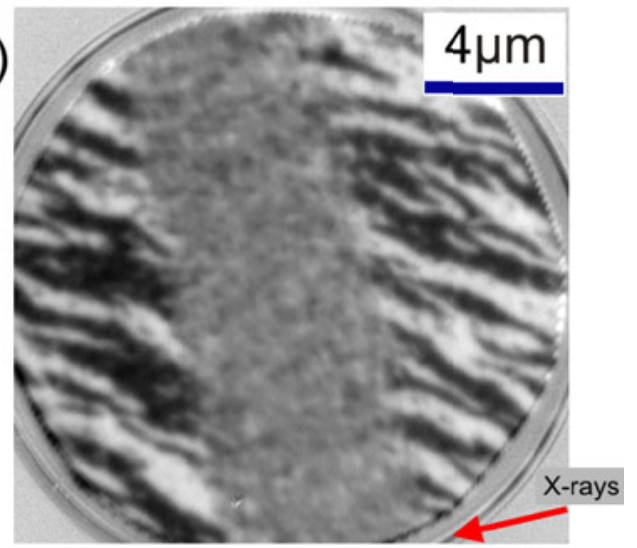

Fig. 1. $\mathrm{Mn} \mathrm{L}_{3}$ edge PEEM-XMCD images of demagnetized (a) $53 \mathrm{~nm}$ thick LSMO/LSAT and (b) $29 \mathrm{~nm}$ thick LSMO/BTO samples. White and black contrast indicates magnetization along the X-ray propagation direction, and this direction is shown by the arrow in the lower right of each image. Gray contrast indicates magnetization oblique to the X-ray propagation direction. The film on LSAT shows fourfold in-plane anisotropy evenly distributed across the field of view, whereas the film on BTO shows a 5 micron wide striped modulation of the magnetic contrast.

strain imposed by alternating $a$-axis domains in the BTO substrate [7].

Although the LSMO/LSAT sample is nearly strainfree, there is a linear dichroism present at the surface of the sample due to the reduction of coordination of the Mn environment. Spectro-microscopy in the same field of view as Figure 1a at grazing incidence using linearly polarized light is plotted in Figure 3 with a significant linear dichrosim at both the $\mathrm{L}_{3}$ and $\mathrm{L}_{2}$ peaks. Comparison of the linear dichroism spectra for the regions labeled A, $\mathrm{B}, \mathrm{C}$, and D in Figure 1a reveals that there is a slight change in the spectra between domains whose magnetization lies almost completely along the X-ray propagation direction and domains whose magnetization is oblique to the X-ray propagation direction (Fig. 3b). Taking the difference of these spectra (Fig. 3c) highlights the magneticonly contribution to the linear dichroism. The magnitude of this magnetic linear dichroism at the $\mathrm{L}_{2}$ edge is approximately $2 \%$ of the maximum X-ray absorption signal, and

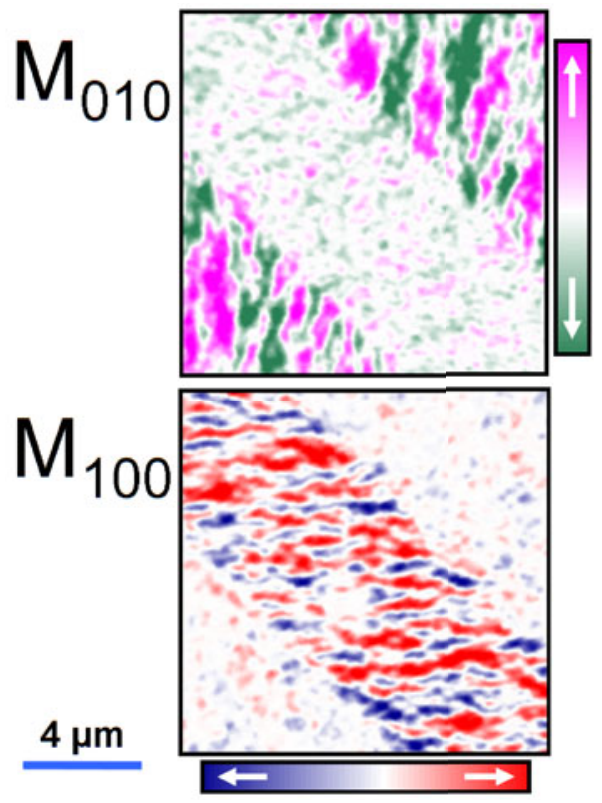

Fig. 2. Vector decomposition of magnetization along $\langle 100\rangle$-type directions for the $29 \mathrm{~nm}$ thick LSMO/BTO sample.

we can estimate that the magnetic contribution is half of the total linear dichroism signal at the $\mathrm{L}_{2}$ edge at room temperature.

We may directly compare the magnetic linear dichroism spectrum extracted from the demagnetized spectromicroscopy experiment to a magnetic linear dichroism spectrum measured in applied field. In normal incidence geometry, there are equivalent $\langle 100\rangle$ axes that we can probe by aligning the electric field component of the linearly polarized X-rays along either the horizontal or vertical directions (e.g. $E \|[100]$ or $E \|[010])$. In zero applied magnetic field for a demagnetized sample, we would average over all ferromagnetic domains and there would be no net difference in absorption. However, application of a $2 \mathrm{~T}$ magnetic field in the plane of the sample along the [100] direction saturates the LSMO film into a single domain state and thus we may probe magnetic-only contribution to the linear dichroism (XMLD). While the XMLD lineshapes for the LSMO/LSAT sample in Figures 3 and 4 are similar, comparison of the XMLD spectra for the $53 \mathrm{~nm}$ LSMO films on LSAT and BTO shown in Figure 4 have qualitatively different lineshapes, illustrating that the average tensile strain affects the magnetic anisotropy of the LSMO film on BTO as compared to the LSAT sample. As the epitaxial strain state imposes a distortion of the local $\mathrm{Mn}$ environment and thus alters the ligand field, a difference in the XMLD spectra can be expected. Indeed, such differences in ligand field due epitaxial strain between LSMO and dielectric substrates has been previously established (e.g. Ref. [10]). To demonstrate the dynamic tuning of film magnetic properties by modulation of strain state via the ferroelectric substrate, we can manipulate the ferroelectric domain population with either temperature or applied electric field. 


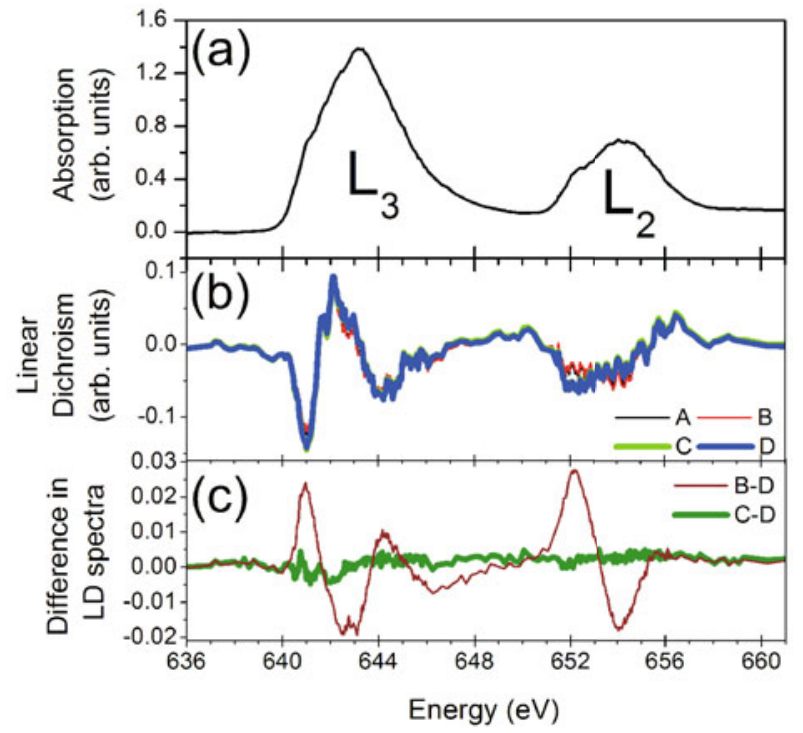

Fig. 3. (a) $\mathrm{Mn} \mathrm{L}_{3}$ polarization averaged X-ray absorption spectrum of $53 \mathrm{~nm}$ thick LSMO/LSAT sample measured via PEEM spectromicroscopy in grazing incidence. (b) A large linear dichroism from both structural and magnetic origins is present when comparing absorption of X-rays with polarization oriented along in-plane versus out-of-plane directions, and (c) the magnetic-only dichroism can be measured by taking the difference of linear dichroism spectra between areas magnetized along orthogonal in-plane directions (e.g. areas B and D) whereas no difference in dichroism signal is seen for areas with antiparallel magnetization (e.g. areas C and D).
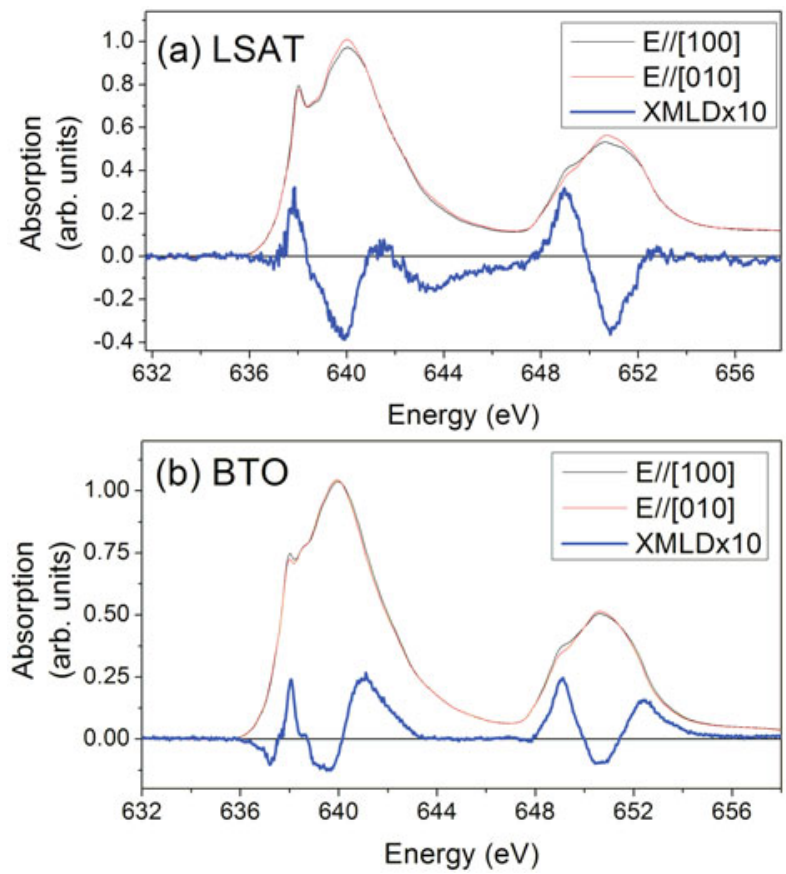

Fig. 4. $\mathrm{Mn}_{3,2} \mathrm{X}$-ray magnetic linear dichroism spectra of the $53 \mathrm{~nm}$ thick (a) LSMO/LSAT and (b) LSMO/BTO samples measured at normal incidence, comparing X-ray absorption along in-plane [100] and [010] directions with $H=2 \mathrm{~T} \|$ [100]. There are strain-induced differences between the XMLD spectra, such as an increase in XMLD intensity near $641 \mathrm{eV}$ and $653 \mathrm{eV}$ for the LSMO/BTO sample.

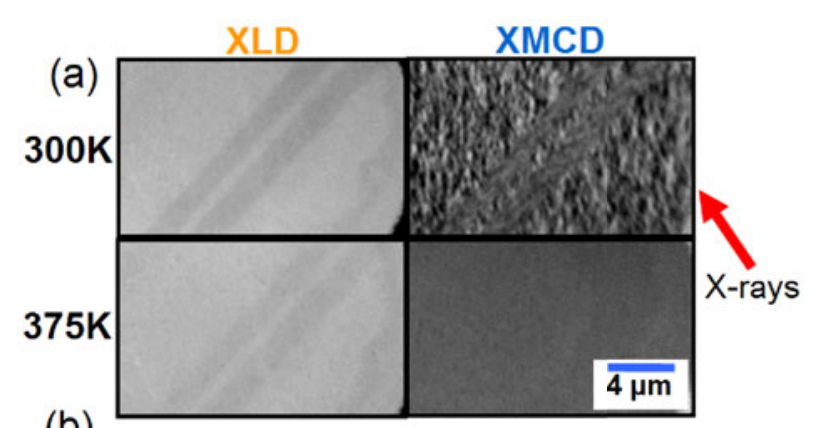

(b)

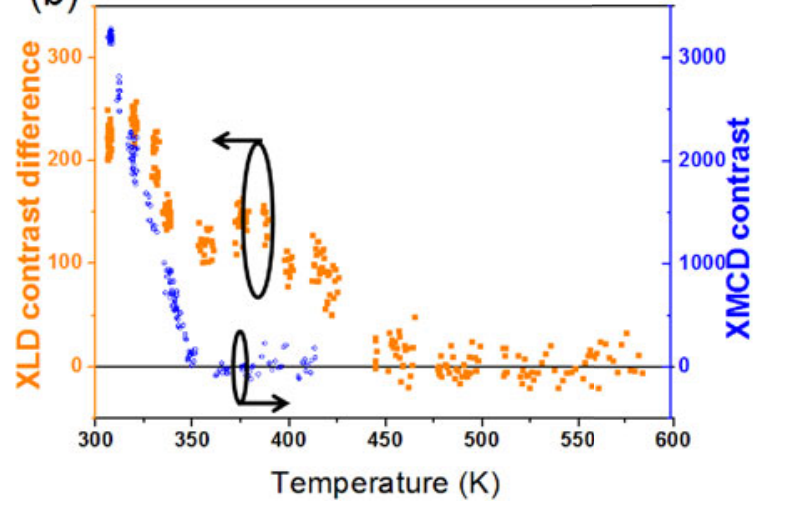

Fig. 5. (a) Images taken in the same area with PEEM-XLD (left) and XMCD (right) for the $29 \mathrm{~nm}$ thick LSMO/BTO sample at room temperature and $375 \mathrm{~K}$. The arrow indicates the $\mathrm{X}$-ray propagation direction. (b) Temperature dependence of XLD and XMCD intensity across the LSMO Curie temperature $(T \approx 350 \mathrm{~K})$ and the BTO tetragonal-cubic phase transition $(T \approx 410 \mathrm{~K})$.

\subsection{Temperature-induced changes in magnetic domain structure}

Temperature-dependent imaging of the LSMO/BTO sample with XMCD and XLD at the Mn L edges (Fig. 5) show that there is a strong temperature dependence of both XMCD and XLD intensity in the temperature range from $300 \mathrm{~K}$ to $450 \mathrm{~K}$. Figure 5a illustrates the spatially resolved change in XMCD and XLD for temperatures below and above the Curie temperature of the LSMO film, while Figure 5b plots the maximum difference in XMCD contrast between white and black domains at the $\mathrm{L}_{3}$ edge peak and difference in XLD contrast at the $\mathrm{L}_{2}$ edge between the two variants of LSMO on $a$-axis BTO domains. The XMCD contrast converges to zero at approximately $350 \mathrm{~K}$, close to the bulk Curie temperature of LSMO $(370 \mathrm{~K})$. The XLD contrast temperature dependence has two steps near $350 \mathrm{~K}$ and $425 \mathrm{~K}$, which are near the Curie temperatures of LSMO and BTO, respectively. In the temperature range between $350 \mathrm{~K}$ and $425 \mathrm{~K}$, the LSMO film is non-magnetic but still experiences the structural distortion imposed by the ferroelectric substrate domain structure. Thus at room temperature there are both magnetic and structural contributions to the linear dichroism, and the persistence of the structural linear dichroism up to the BTO ferroelectric Curie temperature illustrates 


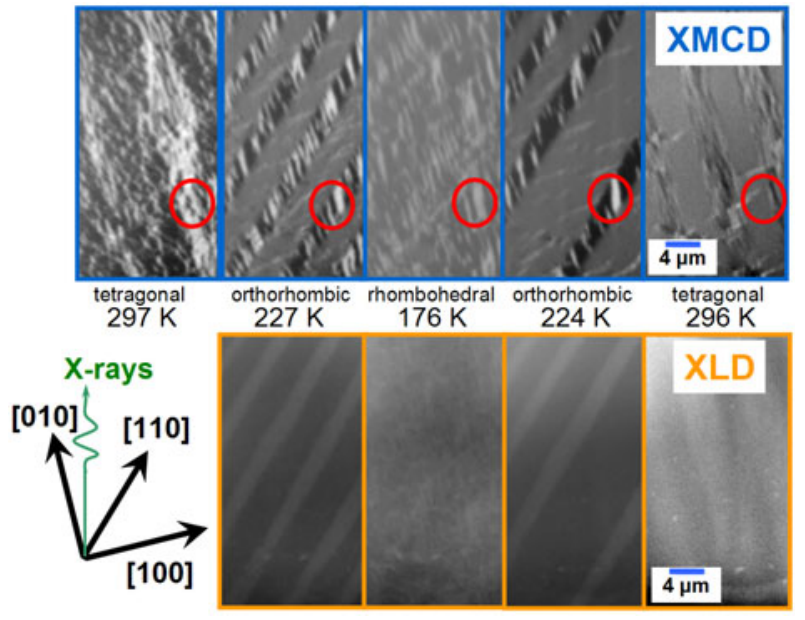

Fig. 6. PEEM-XMCD (top) and XLD (bottom) images for the $53 \mathrm{~nm}$ thick LSMO/BTO sample during a cooling cycle going from room temperature to $160 \mathrm{~K}$ and back. The circled region indicates the same physical location on the LSMO film.

the strong influence that the BTO domain structure has on the local Mn environment.

Similarly, on cooling to the tetragonal-orthorhombic $(\mathrm{T} \approx 290 \mathrm{~K})$ and orthorhombic-rhombohedral $(\mathrm{T} \approx$ $190 \mathrm{~K}$ ) phase transitions of BTO, the BTO domain population changes and both domain wall motion and a change in easy axis of the LSMO film can be seen (Fig. 6). By monitoring the same area of the LSMO film during a temperature cycle from $300 \mathrm{~K}$ to $160 \mathrm{~K}$ to $300 \mathrm{~K}$, we can record the evolution of XMCD and XLD as the sample traverses BTO phase transitions. The original magnetic domain configuration was set by demagnetizing the sample, and illustrates the domain configuration in a region strained by a large $a$-axis BTO domain similar to the regions shown in Figure 1b. The sample is measured at a slight angle away from the [010] direction to measure projections of magnetization along either $\langle 100\rangle$ or $\langle 110\rangle$ directions. By geometry, we expect strong contrast for domains along the [010] axis, an intermediate contrast along the [110] axis, and almost no contrast for domains along either the [100] in-plane axis or [001] out-of-plane axis.

Upon traversing each phase transition in zero applied magnetic field, the original demagnetized domain structure with LSMO domains significantly oriented along the [010] axis as shown at $297 \mathrm{~K}$ changes into a heterogeneous contrast pattern as shown by the XMCD image at $227 \mathrm{~K}$, and the single $a$-axis region of BTO changes into a multidomain configuration as shown by the change in linear dichroism domain pattern in the orthorhombic phase. While the original magnetic domain configuration in the circled region had a strong alignment along the [010] axis, when returning to $296 \mathrm{~K}$ the domains in this region have a neutral contrast, showing that the temperature cycling has produced a $90^{\circ}$ rotation of magnetization for these domains due to reconfiguring the ferroelectric domain structure of the underlying substrate.

The change in XMCD intensity across a single phase transition is plotted in Figure 7. By evaluating the change (a)

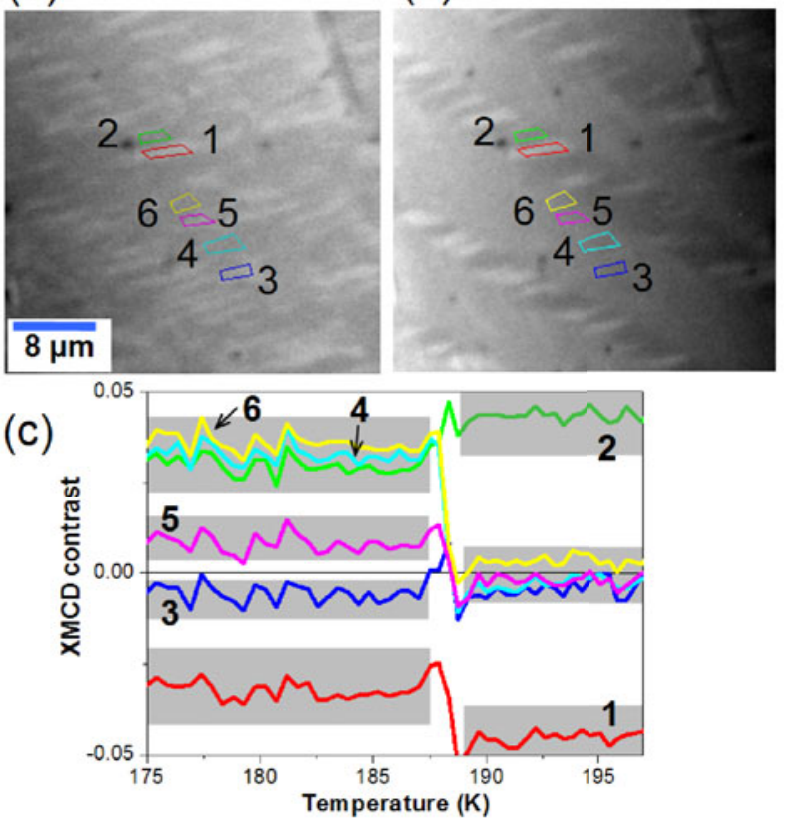

Fig. 7. PEEM single polarization images of the $53 \mathrm{~nm}$ thick LSMO/BTO sample at the $\mathrm{Mn} \mathrm{L}_{3}$ edge at (a) $165 \mathrm{~K}$ (rhombohedral BTO) and (b) $200 \mathrm{~K}$ (orthorhombic BTO), with six example regions selected. The field of view is 36 microns. In panel (c), the XMCD intensity is determined for each region, illustrating a change from four contrast levels below $187 \mathrm{~K}$ to three contrast levels above $187 \mathrm{~K}$.

in XMCD contrast, equivalent to the projection of the magnetization along the beam direction, we see that the XMCD contrast levels strongly change in individual domains from four distinct levels of contrast (fourfold easy axis evenly distributed in the field of view) in the BTO rhombohedral phase to three levels of contrast with areas either strongly aligned along the X-ray direction or oblique to the X-ray direction. In other words, in the orthorhombic phase we have areas with a uniaxial magnetic easy axis either along or oblique to the X-ray direction. Comparison to spatially averaged bulk magnetometry measurements on LSMO/BTO show that a rotation of magnetic anisotropy from $\langle 110\rangle$ type directions to $\langle 100\rangle$ type directions can occur across the BTO phase transition [4]. Thus, a magnetization rotation of either $45^{\circ}$ or $90^{\circ}$ can be generated in the LSMO/BTO system by manipulating the ferroelectric phase and orientation of ferroelectric axis with temperature.

\subsection{Electric field-induced changes in magnetic domain structure}

It is also possible to manipulate the BTO domain population at a given temperature by application of an electric field. In this case, during PEEM imaging we hold the LSMO film at ground potential with respect to the sample holder and apply a voltage pulse to the back of the sample, thus we can apply more than $5 \mathrm{kV} / \mathrm{cm}$ across the BTO 

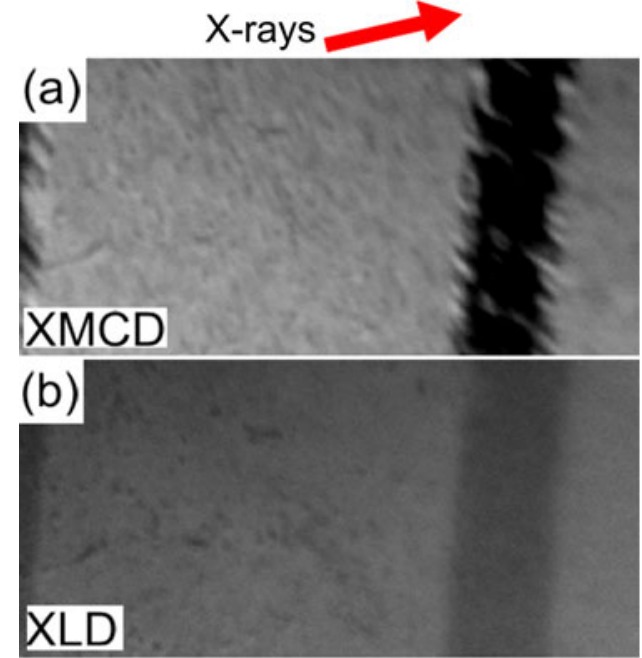

(c)

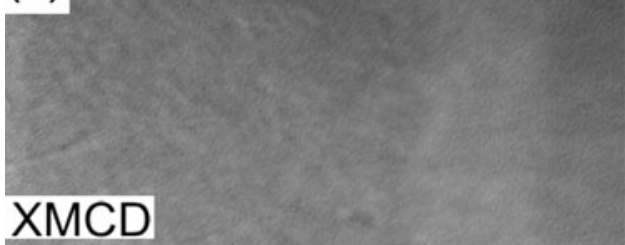

(d)

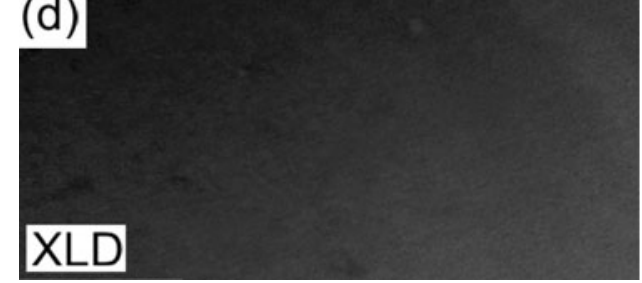

Fig. 8. Mn L edge XMCD and XLD images for the $29 \mathrm{~nm}$ thick LSMO film on BTO before $(\mathrm{a}, \mathrm{b})$ and after $(\mathrm{c}, \mathrm{d})$ a $10 \mathrm{kV} / \mathrm{cm}$ pulse applied across the BTO substrate thickness, with an arrow indicating the X-ray incidence direction. In this field of view, the BTO pre-pulse state has a majority of $a$-axis domains with a 4 micron wide thin stripe of $c$-axis domain with domain walls along the in-plane [010] direction. After the pulse, the change in BTO domain structure as probed with Mn XLD contrast is coincident with rotation of the LSMO magnetization direction in the thin stripe area. The XMCD contrast scale is the same for panels $(\mathrm{a}, \mathrm{c})$ and the XLD contrast scale is the same for panels $(b, d)$.

substrate thickness. Application of a voltage pulse across the thickness of a thermally randomized BTO substrate with both a and c domains (Fig. 8) results in a conversion of a-type domains to c-type domains. In Figure 8, the XLD domain boundaries before poling are along the [010] direction, thus the BTO in this region is a majority of atype domains with a central c-type domain stripe [7]. At the boundary between a- and c-type ferroelectric domains, the LSMO domain structure differs in contrast, with the magnetization of the LSMO on the $c$-axis BTO domain oriented along the X-ray propagation direction and the magnetization of the LSMO on the $a$-axis BTO domain oblique to the $\mathrm{X}$-ray propagation direction.
After a voltage pulse of $10 \mathrm{kV} / \mathrm{cm}$, we can visualize the change in film strain state with XLD in Figure 8d, and see a coincident rotation of magnetization after the pulse with XMCD in Figure 8c. The level of XLD contrast in the $c$-axis region of Figure $8 \mathrm{~b}$ matches the contrast level of the entire field of view for panel (d). Thus the field pulse has converted the $a$-axis regions into $c$-axis regions and we have changed the strain state in those areas. However, we can only distinguish between $a$-axis and $c$-axis regions and cannot differentiate between ' $c+$ ' and ' $c-$ ' orientations of the BTO. Examination of the change in magnetic domain structure reveals that while the $a$-axis regions have a similar XMCD contrast level, the $c$-axis regions have rotated their magnetization to closely match the contrast level of the LSMO in the $a$-axis regions. If the $c$-axis domain region reversed with the application of the voltage pulse, we could expect influence of both charge and strain effects on the magnetization of LSMO. While previous studies have shown that an 11 unit cell thick LSMO film can demonstrate a significant change in properties due to induced charge from an adjacent $\mathrm{Pb}(\mathrm{Zr}, \mathrm{Ti}) \mathrm{O}_{3}$ layer [15], it is unclear if such a similar change in interfacial charge would affect a $29 \mathrm{~nm}$ thick LSMO film. Further work is needed to elucidate the mechanism of this magnetization rotation and the roles that modulation of strain and induced charge play at the LSMO/BTO interface.

In Figure 9, a schematic summarizing the straininduced magnetic domain configurations of LSMO due to the ferroelectric domain configuration of BTO is presented. At room temperature, LSMO coupled to an $a$-axis BTO domain (Fig. 9a) exhibits a uniaxial strain state and a strong in-plane uniaxial magnetic anisotropy, whereas LSMO coupled to $c$-axis BTO (Fig. 9b) has a biaxial strain state and weak fourfold in-plane anisotropy. Above $350 \mathrm{~K}$, LSMO is paramagnetic (Fig. 9c) but a structural distortion in LSMO induced by the BTO is still present. Above $425 \mathrm{~K}$, BTO is paraelectric (Fig. 9d) and the LSMO XLD intensity induced by the BTO ferroelectric domain structure vanishes. For comparison, the dominant contribution to XLD for LSMO grown on LSAT (Fig. 9e) is due to ferromagnetic ordering. As shown experimentally in Figure 8 and schematically in Figure 9, we have demonstrated that by rotating the ferroelectric axis in the BTO substrate with an electric field pulse, we have reoriented the magnetization of the LSMO due to the strong elastic interaction between the film and substrate.

\section{Conclusions}

We have resolved the strain-dependent effect of BTO ferroelectric domains on LSMO magnetic domains, and have measured the temperature dependence of the magnetic anisotropy of LSMO across the BTO phase transitions. There is a strong effect on the LSMO easy axis due to modulation of strain by $a$-axis BTO domains, namely an LSMO easy axis rotation of $45^{\circ}$ when going from the rhombohedral to the orthorhombic BTO phase, and we have measured the effect of reorientation of these BTO domains at the spatial resolution of a single LSMO domain. 
(a)

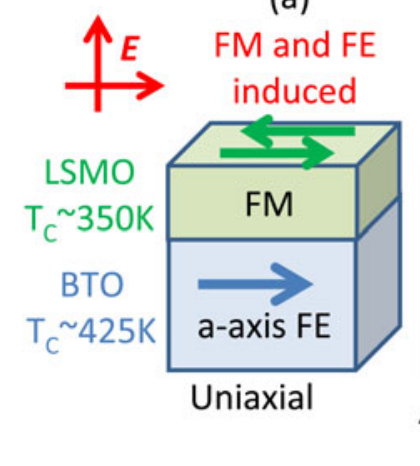

(b) FM and FE

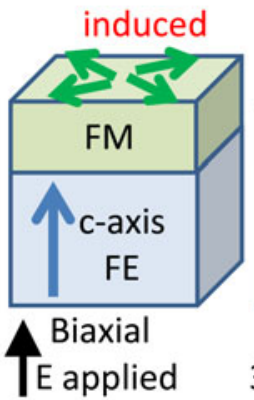

(c)

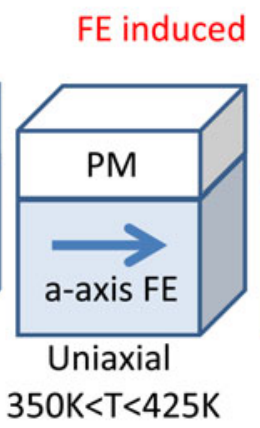

(d)

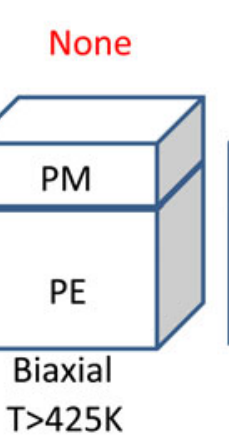

(e)

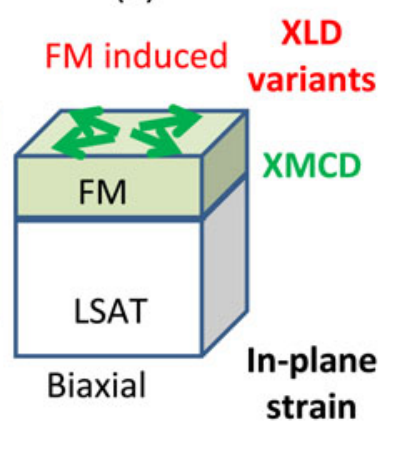

Fig. 9. Magnetic configurations and contributions to the linear dichroism in the LSMO/BTO system at room temperature (a, b), and at elevated temperatures (c, d). The LSMO/LSAT sample at room temperature is shown (e) for comparison.

Thus with such a strong elastic coupling between film and substrate and the ability to dynamically tune the strain state of the film with reorientation of the BTO ferroelectric axis, we show that this model artificial multiferroic can hold promise for room-temperature magneto-electric coupling at the nanoscale, and furthermore shows a possible materials system to be employed in sub-micron devices that exploit electric field control of magnetic anisotropy.

The authors would like to thank C.W. Schneider for the use of his X-ray diffractometer, and L. Le Guyader and M. Buzzi for assistance with image analysis software. Part of this work was performed at the Swiss Light Source, Paul Scherrer Institute, Villigen, Switzerland. The Advanced Light Source is supported by the Director, Office of Science, Office of Basic Energy Sciences, of the U.S. Department of Energy under Contract No. DE-AC02-05CH11231. This work was partially funded by EU's 7th Framework Program IFOX (NMP3-LA-2010 246102).

\section{References}

1. C.A.F. Vaz, J. Hoffman, C.H. Ahn, R. Ramesh, Adv. Mater. 22, 2900 (2010)

2. R. Datta, S. Kanuri, S.V. Karthik, D. Mazumdar, J.X. Ma, A. Gupta, Appl. Phys. Lett. 97, 071907 (2010)
3. F. Tsui, M.C. Smoak, T.K. Nath, C.B. Eom, Appl. Phys. Lett. 76, 2421 (2000)

4. M.K. Lee, T.K. Nath, C.B. Eom, M.C. Smoak, F. Tsui, Appl. Phys. Lett. 77, 3547 (2000)

5. D. Dale, A. Fleet, J.D. Brock, Y. Suzuki, Appl. Phys. Lett. 82, 3725 (2003)

6. W. Eerenstein, M. Wiora, J.L. Prieto, J.F. Scott, N.D. Mathur, Nat. Mater. 6, 348 (2007)

7. R.V. Chopdekar et al., Phys. Rev. B 86, 014408 (2012)

8. A. Tebano et al., Phys. Rev. Lett. 100, 137401 (2008)

9. M. Huijben, L.W. Martin, Y.H. Chu, M.B. Holcomb, P. Yu, G. Rijnders, D.H.A. Blank, R. Ramesh, Phys. Rev. B 78, 094413 (2008)

10. C. Aruta, G. Ghiringhelli, V. Bisogni, L. Braicovich, N.B. Brookes, A. Tebano, G. Balestrino, Phys. Rev. B 80, 014431 (2009)

11. R.V. Chopdekar, E. Arenholz, Y. Suzuki, Phys. Rev. B 79, 104417 (2009)

12. U. Flechsig, F. Nolting, A.F. Rodriguez, J. Krempasky, C. Quitmann, T. Schmidt, S. Spielmann, D. Zimoch, Am. Inst. Phys. Confer. Proc. 1234, 319 (2010)

13. C. Piamonteze et al., J. Synchr. Rad. 19, 661 (2012)

14. J. Stöhr, H.A. Padmore, S. Anders, T. Stammler, M.R. Scheinfein, Surf. Rev. Lett. 5, 1297 (1998)

15. C.A.F. Vaz, J. Hoffman, Y. Segal, M.S.J. Marshall, J.W. Reiner, Z. Zhang, R.D. Grober, F.J. Walker, C.H. Ahn, J. Appl. Phys. 109, 07D905 (2011) 